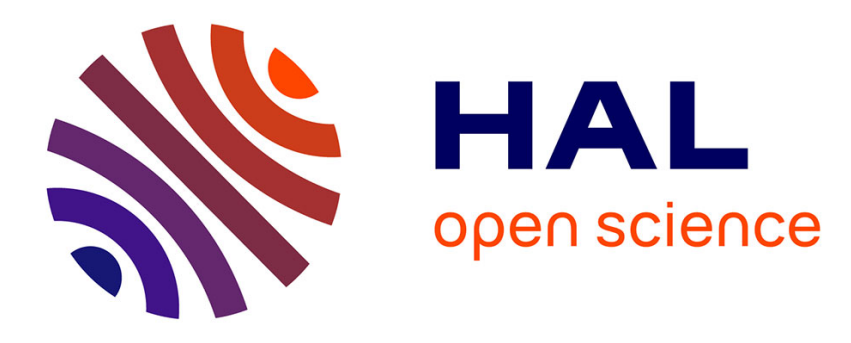

\title{
Les barèmes, outils d'aide à la décision pour les justiciables et les juges
}

Cécile Bourreau-Dubois, Bruno Deffains, Myriam Doriat-Duban, Bruno

Jeandidier

\section{- To cite this version:}

Cécile Bourreau-Dubois, Bruno Deffains, Myriam Doriat-Duban, Bruno Jeandidier. Les barèmes, outils d'aide à la décision pour les justiciables et les juges. Revue d'Economie Politique, 2021, 131 (2021/2), pp.199-222. 10.3917/redp.312.0031 . hal-03054417

\section{HAL Id: hal-03054417 \\ https://hal.univ-lorraine.fr/hal-03054417}

Submitted on 6 Jan 2022

HAL is a multi-disciplinary open access archive for the deposit and dissemination of scientific research documents, whether they are published or not. The documents may come from teaching and research institutions in France or abroad, or from public or private research centers.
L'archive ouverte pluridisciplinaire $\mathbf{H A L}$, est destinée au dépôt et à la diffusion de documents scientifiques de niveau recherche, publiés ou non, émanant des établissements d'enseignement et de recherche français ou étrangers, des laboratoires publics ou privés.

$$
\text { Copyright }
$$




\title{
Les barèmes, outils d'aide à la décision pour les justiciables et les juges ${ }^{1}$
}

\author{
C. Bourreau-Dubois ${ }^{2}$, B. Deffains ${ }^{3}$, M. Doriat-Duban ${ }^{4}$, B. Jeandidier ${ }^{5}$
}

MOTS CLES : Barème - décision de justice - négociation - juge

\section{JEL : K40, K41, K15}

\section{Résumé :}

Dans un contexte français marqué par le développement des barèmes judiciaires, cet article propose une analyse économique de ces derniers, centrée sur les acteurs du litige : juges et justiciables. Après avoir présenté les différentes formes que pouvait prendre un barème judiciaire, l'article, qui prend appui sur une revue de littérature, met en évidence que les barèmes peuvent être envisagés comme des outils d'aide à la décision qui, en fournissant de l'information aux justiciables et en cadrant (plus ou moins fortement selon leur nature) le pouvoir discrétionnaire des juges, jouent un double rôle : incitatif et normatif.

\section{Guidelines: decision-making tools for litigants and judges}

Keywords: Guidelines, legal decision-making, negotiation, judge

\section{JEL : K40, K41, K15}

\section{Abstract:}

In a French context characterized by the development of judicial guidelines, we propose a review of the economic literature on guidelines focusing on the actors of litigation: judges and litigants. We identify two functions of guidelines, considered as decision-making tools that provide information to litigants and frame the discretionary power of judges (more or less strongly depending on their nature): incentive and normative.

\footnotetext{
${ }^{1}$ Cet article est issu d'une recherche qui a bénéficié du soutien de la Mission recherche Droit et Justice (Bourreau-Dubois et al., 2019).

${ }^{2}$ Université de Lorraine, Université de Strasbourg, CNRS, BETA, 54000, Nancy, France (cecile.dubois@univlorraine.fr)

${ }^{3}$ Université Paris 2, CRED, 75000, Paris, France (bruno.deffains@u-paris2.fr)

${ }^{4}$ Université de Lorraine, Université de Strasbourg, CNRS, BETA, 54000, Nancy, France (myriam.duban@univlorraine.fr)

5 Université de Lorraine, Université de Strasbourg, CNRS, BETA, 54000, Nancy, France (bruno.jeandidier@univ-lorraine.fr)
} 


\section{INTRODUCTION}

L'ordonnance du 22 septembre 2017, qui introduit dans le droit français un barème impératif d'indemnisation des licenciements jugés sans cause réelle et sérieuse par le Conseil de prud'hommes, a fait couler beaucoup d'encre dans la communauté des acteurs du droit (magistrats, avocats... $)^{6}$, beaucoup moins chez les économistes ${ }^{7}$ et les acteurs des politiques publiques.

De manière plus générale, les critiques des juristes à l'égard des barèmes portent sur la perte de pouvoir discrétionnaire des juges, c'est-à-dire leur capacité à juger en tenant compte des spécificités de l'affaire mais aussi de leur sensibilité. Du côté des avocats, l'existence de barèmes est considérée comme étant de nature à réduire leur éventail de stratégies dans la procédure contradictoire. Si les barèmes sont moins l'objet de polémiques chez les économistes, c'est sans doute parce que, pour eux, un barème consiste en un outil de politique publique visant, assez classiquement, des objectifs qui peuvent être appréhendés en termes d'efficacité et d'équité. En effet, les barèmes répondent à un objectif d'efficacité lorsqu'ils participent à la maximisation du bien-être social en minimisant, par exemple, le coût social des conflits (réduction de la durée moyenne des affaires, réduction de l'incertitude qui faciliterait l'accord amiable entre défendeur et demandeur) ou celui de la délinquance (augmentation du caractère dissuasif des peines par un relèvement de celles-ci) ${ }^{8}$. Ils poursuivent également des objectifs en termes d'équité, à la fois horizontale et verticale. Dans le premier cas, il s'agit d'assurer une égalité de traitement entre justiciables de caractéristiques équivalentes (équité inter-affaires), voire une équité de traitement des parties impliquées dans une même affaire (équité intra-affaire). Dans le second cas, l'équité verticale correspond à la recherche d'un juste équilibre entre le montant prévu par le barème et l'objet de la décision judiciaire.

\footnotetext{
${ }^{6}$ Cf. article du Monde du 8 janvier 2019, « Prud'hommes : les juges font de la résistance » ou tribune publiée par un collectif d'avocats et de magistrats dans le Monde du 19 décembre 2018 «Plafonnement des indemnités de licenciement : Les juges ne sont pas des ignorants qu'il faudrait remettre dans le droit chemin ».

${ }^{7} \mathrm{Cf}$. étude récente sur la barémisation du contentieux prud'homal par Desrieux, Espinosa (2020).

${ }^{8}$ Dans le cas du barème français d'indemnisation en matière de licenciement, la recherche d'efficacité faisait partie des arguments de ses promoteurs. En fixant un plancher et un plafond d'indemnités, ce barème permet de les contenir en-deçà d'un certain seuil et d'en limiter la variabilité afin que les entreprises puissent plus facilement anticiper, voire provisionner, les charges liées à ce type de contentieux. L'objectif est également de faciliter la négociation entre les parties en cadrant les décisions pour réduire leur variabilité (Barthélémy et Cette, 2015).
} 
Face au débat lancé par les juristes, dans un contexte d'instauration accélérée de barèmes judiciaires en France ${ }^{9}$, il nous semble particulièrement intéressant, de recourir à l'approche économique pour enrichir la réflexion publique relative aux enjeux de la barémisation de la justice. A cette fin, nous proposons de nous appuyer sur la littérature économique disponible pour développer une analyse centrée sur les acteurs du litige et où les barèmes seront considérés comme des outils d'aide à la décision. En effet, en fournissant de l'information aux justiciables et en encadrant (plus ou moins selon leur nature) le pouvoir discrétionnaire du juge, les barèmes peuvent être appréhendés comme des outils d'aide à la décision, jouant un double rôle : incitatif et normatif. Ce faisant nous adoptons une grille de lecture mettant l'accent sur l'impact décisionnel des barèmes sur les justiciables comme sur les juges. Dans une approche d'inspiration néo-classique, par exemple posnérienne ou béckerienne, où tous les agents sont parfaitement rationnels, les barèmes vont constituer une source d'information qui va permettre aux agents de faire les «bonnes anticipations » et, en particulier, aux justiciables de mieux appréhender la décision judiciaire. D'autres grilles d'analyse peuvent également être mobilisées, notamment celle de la rationalité imparfaite. Ainsi, dans une approche à la Sunstein fondée sur la rationalité limitée, et si l'on reprend l'analyse de Ferey et Deffains (2014) en l'appliquant à notre sujet, les barèmes vont permettre d'opérer un redressement cognitif qui peut avoir trois fonctions: limiter les biais cognitifs (biais d'optimisme ou de disponibilité des justiciables que l'on peut observer en matière de conflits), éviter l'exploitation par certains de biais cognitifs à leur avantage (en particulier dans les conflits où les asymétries d'information sont fréquentes), et éviter que les autorités judiciaires soient elles-mêmes victimes de biais. Toujours selon cette même approche mais poussée à l'extrême, les barèmes pourraient aussi s'intégrer dans une démarche de paternalisme libéral : en admettant que les agents sont victimes de biais cognitifs, l'encadrement de leurs décisions par un barème pourrait les rendre plus conformes aux préférences sociales, à condition que le barème reflète effectivement celles-ci.

La première section de cet article vise à montrer que les barèmes recouvrent en réalité des outils d'aide à la décision pouvant prendre des formes très variées. La deuxième et la troisième sections proposent un examen analytique des travaux économiques portant sur les barèmes. Ainsi, la deuxième section permet de souligner le rôle incitatif du barème. Celui-ci est source d'incitations pour les acteurs du conflit, en amont comme en aval du contentieux.

\footnotetext{
${ }^{9}$ Une récente étude de Sayn et al. (2019) dénombre, sans prétendre à l'exhaustivité, 219 outils d'aide à la décision en usage dans les différentes juridictions françaises, outils que l'on peut considérer comme étant des barèmes.
} 
En amont, le barème influence les décisions des justiciables en ce sens qu'il leur fournit des informations permettant de mieux anticiper les jugements auxquels ils sont exposés et peut les inciter à recourir à un mode alternatif de règlement des conflits. En aval, un barème affecte l'activité décisionnaire des juges parce qu'en contribuant à expliciter la norme juridique, il peut inciter ces derniers à adopter des stratégies de contournement pour préserver leur pouvoir discrétionnaire. Enfin, la dernière section, repose sur l'idée que le barème peut jouer un rôle normatif au sens où celui-ci va cadrer les décisions des juges, avec en arrière-plan, les théories de l'interprétation qui distinguent deux approches (Ferey et Deffains, 2014) : l'approche réaliste où l'interprétation de la norme légale est perçue comme un acte de volonté et l'approche formaliste où l'interprétation est davantage perçue comme acte de connaissance. Dans cette perspective, on peut considérer qu'un barème, en particulier lorsqu'il est impératif ( $v s$ indicatif) et surtout s'il fixe une valeur précise plutôt qu'un intervalle de décision («fourchette »), devient l'expression concrète du mythe formaliste : la décision s'impose au juge et donne la primauté à une interprétation de la norme en réduisant potentiellement à néant toute marge de manœuvre en faveur d'interprétations alternatives. L'examen de la littérature permet de montrer que le barème ne suffit cependant pas à ramener les décisions des juges à un acte de connaissance pure et que celles-ci relèvent toujours, même partiellement, d'un acte de volonté, soit parce que les préférences des juges continuent de s'exprimer (juge à la Posner), soit parce que des biais cognitifs subsistent (juge à la Sunstein).

\section{Le barème : un outil d'aide à la décision protéiforme}

Selon Sayn et al. (2019), en France, le terme «barème » est le plus souvent utilisé lorsque l'outil croise quelques critères numériques ou qualitatifs pour proposer une décision exprimée selon une valeur numérique (montant d'indemnité, durée d'emprisonnement, montant d'amende, durée de suspension de permis de conduire...). Les termes « lignes directrices » ou «référentiel» sont plus souvent utilisés lorsque l'outil d'aide à la décision consiste à construire un arbre de décision (éventuellement sous forme de tableau) à partir de quelques critères avec pour finalité d'orienter vers une décision qualitative (condamner ou non; accorder ou non l'autorité parentale; assortir ou non la sanction d'une obligation thérapeutique...) qui peut être une décision judiciaire à proprement parler ou une décision 
d'orientation de procédure ${ }^{10}$. Mais dans les deux cas, l'outil a la même finalité : proposer une décision à partir d'un ensemble de critères restreint.

Si la littérature anglo-saxonne ne fait pas de distinction sémantique selon le type d'outil et recourt indifféremment au terme guidelines, à l'inverse, les juristes français semblent beaucoup plus sensibles au choix du vocabulaire. Par exemple, dans une enquête qualitative auprès de magistrats, plusieurs d'entre eux soulignent qu'il est important de recourir au terme « table de référence » et non pas au terme de «barème » lorsque l'on parle de l'outil d'aide à la décision relatif aux pensions alimentaires pour enfant en cas de séparations parentales, et ce parce que le premier terme expliciterait plus nettement le caractère indicatif (par opposition à impératif) de l'outil (Jeandidier et Ray, 2019) ${ }^{11}$.

D'un outil à l'autre, la force de la proposition est variable car il existe tout un continuum dans le degré de contrainte produit par ces outils, ce qui conduit à ce que la potentialité de perte de pouvoir discrétionnaire pour le juge soit plus ou moins importante. Ce continuum a plusieurs origines : le type de propositions issues du barème (selon les cas, ce dernier indiquera une valeur unique, une valeur minimale, une valeur maximale, une fourchette), l'indication d'usage (barème strictement impératif, barème ayant valeur de présomption avec faculté pour le juge d'y déroger de manière argumentée, barème indicatif, qui peut ou non être l'unique motivation de la décision...), la place dans la hiérarchie des normes (barème inclus dans la loi, une ordonnance, un décret, un arrêté, une circulaire, voire en usage dans la profession sans aucune assise légale). L'acceptabilité de ces outils d'aide à la décision par le milieu judiciaire repose également sur leur légitimité perçue. A cet égard, on peut distinguer les barèmes construits des barèmes constatés. Les premiers sont conçus à partir d'un ensemble de choix, reposant sur des raisonnements juridiques, économiques et démographiques ou autres (qui sont toujours discutables), d'un nombre restreint de critères, à partir desquels on peut construire des catégories homogènes. Les seconds, à l'aide de différentes méthodes statistiques notamment, cherchent simplement à reproduire, sans raisonnements particuliers,

\footnotetext{
${ }^{10}$ Par exemple, dans les lignes directrices sur l'usage et la détention en vue d'usage de produits stupéfiants, selon différents critères (type de produit, quantité détenue, mineur/majeur, degré de récidive) le barème prévoit soit un simple rappel à la loi sans garde à vue (ce qui constitue une décision judiciaire finale en soi), soit une garde à vue avec comparution immédiate ou encore une convocation par un officier de police judiciaire (dans ces deux cas, il s'agit d'une décision de procédure qui renvoie à plus tard la décision d'éventuelle sanction, mais on peut penser que la décision de procédure peut avoir une incidence sur la décision judiciaire finale).

${ }^{11}$ De même on peut souligner que dans le cas de l'indemnisation des préjudices corporels ce sont des référentiels qui sont utilisés (ex : nomenclature DINTHILAC) par les fonds d'indemnisation comme l'ONIAM ou le FIVA voire par les cours d'appel, dans un contexte où il n'existe pas de barème officiel mis en place de manière réglementaire (Porchy-Simon, 2014).
} 
les décisions passées prises par les acteurs du droit ${ }^{12}$. Face à cette diversité des caractéristiques des outils, dans cet article nous ferons nôtre la définition englobante avancée par Sayn et al. (2019) selon lesquels un barème est un outil d'aide à la décision «permettant de préciser des critères de décision fixés par le droit applicable, en resserrant le maillage normatif et parfois en écartant corrélativement d'autres critères qui auraient pu être retenus » (p. 7).

\section{Barèmes et décisions des acteurs du conflit : le rôle incitatif du barème}

Le barème, en tant qu'outil d'aide à la décision, peut être mobilisé par les justiciables comme par les juges. Pour les premiers, il s'agit de voir dans quelle mesure un barème, en fournissant de l'information leur permettant de mieux anticiper le jugement, va faciliter ou au contraire décourager le règlement amiable du conflit (3.1). Du côté des juges, la question est plutôt de savoir dans quelle mesure la présence d'un barème, qui revient à expliciter la norme juridique et donc à restreindre la marge d'interprétation des juges, incite ces derniers à adopter des stratégies destinées à faire prévaloir leurs préférences personnelles (3.2).

\subsection{Le barème, facilitateur ou obstacle à la négociation des parties}

On peut penser qu'en l'absence de barème, les attentes des parties tendraient plus facilement à diverger, le jugement étant plus incertain, tandis que leur introduction faciliterait la coopération entre les parties, désormais plus à même d'anticiper la décision du juge. L'objectif de favoriser le règlement amiable des litiges peut conduire à estimer qu'il serait alors utile de limiter le pouvoir discrétionnaire des juges pour faciliter la coopération. Mais on peut également imaginer que, si les parties manifestent de l'aversion pour le risque ou sont plutôt pessimistes sur le jugement attendu, plus l'incertitude sur le jugement est grande et plus elles vont être incitées à négocier pour échapper au risque du procès, rendant alors inutile l'instauration d'un barème. Dès lors, la question se pose de savoir si les barèmes, en réduisant l'incertitude, incitent ou au contraire désincitent à la coopération.

Deffains et Langlais (2006) apportent une réponse en comparant l'impact des barèmes indicatifs et impératifs sur la coopération des parents, en ce qui concerne la pension alimentaire en cas de divorce. Un barème indicatif laisse une marge de manœuvre au juge qui peut décider de s'en écarter (il reste donc une marge d'incertitude) tandis qu'un barème

\footnotetext{
${ }^{12}$ On peut trouver de nombreux exemples de barèmes en usage en France illustrant notre propos dans Sayn et al. (2019) aux pages 387 à 691 .
} 
impératif contraint le juge dans sa décision (la règle de calcul est connue donc la décision est prévisible). Les auteurs construisent un modèle théorique de décision où ils introduisent une asymétrie d'information entre les parents et le juge. Leur résultat contredit l'idée selon laquelle plus de certitude permet plus de négociations. En effet, ils montrent que c'est au contraire l'incertitude sur la décision des juges qui va inciter les parents à négocier, s'ils ont de l'aversion pour le risque ou sont pessimistes, car l'arrangement permet à ce type de parents d'échapper au risque. En conséquence, un barème indicatif (risque) est préférable à un barème impératif (certitude) si l'on souhaite favoriser la coopération dès lors que les parties ont de l'aversion pour le risque ou sont pessimistes. L'introduction de barèmes indicatifs serait donc préférable à des barèmes impératifs si l'objectif poursuivi est d'accroître la coopération entre les parties.

Plus largement, le modèle de Deffains et Langlais (2006) interroge sur le fait de savoir si c'est le risque qui incite les parties à négocier ou si c'est au contraire la certitude qui permet l'arrangement. En d'autres termes, la certitude qu'engendre le barème surtout lorsqu'il est impératif, au lieu de servir de référence à l'accord, pourrait nuire à l'arrangement car l'élément déterminant d'un accord amiable serait plutôt la volonté des parties d'échapper au risque que celle d'anticiper correctement le jugement. Les travaux menés sur la procédure de plaider coupable en matière criminelle permettent d'obtenir un éclairage intéressant sur cette question. Les modèles de plaider coupable avancent généralement l'hypothèse que c'est le risque du procès qui va inciter l'accusé à plaider coupable afin d'obtenir une peine certaine plutôt que de risquer un jugement aléatoire (Ancelot et Doriat-Duban, 2010). En conséquence, un barème, parce qu'il rend le jugement plus prévisible, devrait désinciter l'accusé à plaider coupable.

Taha (2001) s'est intéressé à cette question et propose une analyse empirique sur la base d'affaires pénales terminées et issues de l'Administrative Office of the U.S. Courts. Il montre que le taux de plaider coupable augmente après l'instauration des barèmes, contrairement aux prévisions des modèles théoriques fondés sur l'aversion au risque (c'est la peur du risque qui incite à plaider coupable) ou de signal (les innocents refusent de plaider coupable et se «signalent » ainsi comme innocents). Il n'est cependant pas certain que plus de certitude (et donc moins de risque) conduise à plus d'accords. Poursuivant ses investigations, il ajoute qu'en réalité l'augmentation du taux d'accord est liée au fait que les procureurs, après l'instauration des lignes directrices, ont réduit les charges dans l'étape préalable aux poursuites devant le juge. Autrement dit, les accusés plaident davantage coupables parce que 
les procureurs, en accord avec les avocats de la défense, diminuent les charges retenues contre eux pour favoriser l'arrangement; le barème est donc contourné. Ce n'est donc pas l'effet «certitude » qui accroît les incitations à négocier mais la «manipulation » du barème par les procureurs pour conduire à des peines plus faibles. La négociation est donc bien encouragée par la certitude, mais la certitude d'avoir une peine réduite basée sur des charges atténuées de sorte à pouvoir se situer sur la partie du barème correspondant à des peines plus faibles.

Un éclairage théorique peut également être apporté dans le cas du plaider-coupable américain. Reinganum (2000) montre en effet, par un modèle « de signal », que la probabilité de plaider coupable ne devrait pas être influencée par l'introduction d'un barème. L'auteure propose plus précisément un modèle de théorie des jeux dans lequel une commission de jugement peut ex ante imposer l'application du barème ou au contraire s'en remettre à la décision du juge lorsque la procédure de plaider-coupable échoue. Dans ce modèle, l'asymétrie d'information porte sur le degré de gravité du crime, connu seulement du criminel. A l'inverse, le procureur connaît uniquement la distribution a priori de la gravité et des caractéristiques du délinquant ainsi que la distribution a priori des peines en cas de jugement. Le pouvoir de négociation est donc dans les mains de l'accusé puisque c'est lui qui fait l'offre que le procureur accepte ou rejette. En cas de refus, l'affaire est portée devant le tribunal qui est supposé recevoir un «signal» qui peut être «informatif» ou «non-informatif» sur la gravité du cas et les caractéristiques de l'accusé. S'il l'est, le tribunal, qu'il soit soumis à un barème ou libre de sa décision, impose la sanction «idéale » correspondant à ce type d'affaires. S'il ne l'est pas, la sanction diffère selon le régime choisi ex ante par la commission : barème ou discrétion du juge. Au final, trois résultats théoriques sont obtenus. D'abord, la proposition de l'accusé est une fonction croissante de la gravité du crime et de la sanction anticipée si le signal est «noninformatif » (sanction qui correspond soit au barème, soit à la sanction anticipée du juge selon l'option choisie par la commission) de sorte qu'un accusé qui anticipe une sanction plus élevée formulera une proposition également plus élevée. Ensuite, la probabilité de procès à l'équilibre est une fonction décroissante de la vraie gravité du crime et est indépendante de la sanction anticipée ; en d'autres termes, les affaires de gravité supérieure ont plus de chances d'être résolues par un plaider-coupable. Enfin, la sentence optimale telle que spécifiée dans le barème est supérieure à la sentence optimale du tribunal lorsque la sentence est laissée à la discrétion du juge. Cela s'explique par des questions de temporalité et des problèmes d'asymétrie d'information : la commission décide ex ante, avant le début de la procédure de plaider-coupable, de laisser ou non la liberté au juge dans le prononcé du jugement; elle doit 
donc décider de la procédure avant que l'information ne soit disponible puisqu'elle est révélée par la procédure de plaider coupable qui opère une sélection parmi les accusés, ceux ayant commis les délits plus graves étant davantage incités à plaider-coupable. Pour l'auteure, ces résultats sont conformes à l'observation, notamment le second résultat qui implique que la probabilité attendue de procès est indépendante de l'option choisie par la commission, ce qui est compatible avec le fait que le barème n'a pas d'influence sur le taux de plaider-coupable des accusés. En d'autres termes, dans le cas présent, le barème n'aurait pas d'influence sur la négociation.

L'analyse économique conduit finalement à des résultats assez mitigés quant à l'impact des barèmes sur le règlement amiable des litiges ${ }^{13}$. Il n'est en particulier pas du tout certain que les barèmes facilitent la négociation entre les parties ${ }^{14}$. En définitive, avec ou sans barèmes, de nombreux litiges vont continuer à aller devant les juges. La question est alors celle de l'impact du barème sur la façon dont les juges envisagent leur activité professionnelle.

\subsection{Le barème, générateur de comportements stratégiques parmi les acteurs du système juridique}

L'instauration de barèmes, en particulier dans un système de hiérarchie juridique où interviennent plusieurs acteurs, peut modifier la hiérarchie des pouvoirs entre acteurs, et plus particulièrement réduire celui des juges (3.2.1). Face à l'instauration de barèmes et à une potentielle perte de pouvoir discrétionnaire, les juges vont développer différents comportements. Ils peuvent plus précisément résister ou s'adapter stratégiquement (3.2.2).

\subsubsection{Substitution de pouvoir entre acteurs du système judiciaire}

La mise en œuvre d'un barème conduit à modifier les jeux de pouvoir, notamment entre juge et procureur, mais aussi entre législateur et juges, censés appliquer les décisions dans le sens souhaité par le législateur.

L'article de Taha (2001) porte plus spécifiquement sur les conflits de pouvoir entre le procureur et le juge. L'auteur distingue deux phases de négociation dans le cadre du plaider-

\footnotetext{
${ }^{13}$ Résultats d'autant plus nuancés que les enjeux qui sont au cœur de la négociation dans les articles cités ne sont pas de même nature : un montant de pension alimentaire en droit de la famille, une peine de prison en droit pénal.

${ }^{14}$ Le barème peut même encourager l'émergence des conflits. C'est l'idée défendue par Allen et Brinig (2012) en prenant l'exemple des barèmes en matière de fixation de pension alimentaire. Selon eux, lorsque ces barèmes sont conçus de telle sorte qu'ils conduisent à un transfert de richesse trop favorable aux parents gardiens, ils pourraient inciter le parent susceptible d'obtenir la résidence de l'enfant à demander le divorce.
} 
coupable : avant et après la mise en accusation. Une fois la mise en accusation effectuée, le barème contraint la négociation du plaider-coupable donc réduit le pouvoir de négociation du procureur. Les procureurs/avocats de la défense vont donc agir ex ante, avant la définition des charges qui vont être retenues. La contrepartie d'une fréquence accrue de plaider-coupable serait donc la réduction des charges par les procureurs qui usent ainsi de leur pouvoir discrétionnaire et maintiennent par ce biais leur rôle central dans le mode de résolution des affaires.

Miceli (2007) pose quant à lui la question de l'interaction entre le législateur et les juges dans un processus séquentiel de décision, le législateur et les juges agissant à des moments différents (ex ante pour le législateur, ex post pour le juge) et ayant des opinions potentiellement divergentes sur la fonction sociale de la punition. Selon le modèle standard d'économie du crime proposé par Becker (1968), l'objectif premier de la sanction est la dissuasion. Les sanctions sont donc définies dans le but de dissuader les délinquants potentiels, qui valorisent l'acte illégal davantage que le préjudice social qu'il induit. Les peines sont alors indépendantes des caractéristiques du délinquant et reflètent plutôt le coût social de l'acte illégal dans une perspective d'internalisation (aligner les coûts privés du délinquant sur le coût social de son acte). Mais d'autres éléments, notamment relatifs à l'équité (caractéristiques du délinquant, de l'affaire), peuvent également être intégrés dans la définition des peines. Le législateur semble mieux placé pour poursuivre l'objectif de dissuasion par la définition de sanctions ex ante, indépendantes des caractéristiques spécifiques du prévenu et de l'affaire, parce qu'elles vont servir de signaux aux délinquants potentiels pour les dissuader de réaliser certains actes illégaux. En revanche, les juges sont mieux à même de poursuivre l'objectif d'équité ex post parce qu'ils sont seuls en mesure de prendre en compte les circonstances particulières de l'affaire qu'ils ont à juger. Les barèmes peuvent alors réaliser un équilibre entre ces valeurs sociales concurrentes.

Pour Miceli (2007), étudier l'interaction entre le législateur et les juges dans la détermination des sanctions pénales revient à déterminer le degré optimal de discrétion judiciaire, en se focalisant sur les deux objectifs sociaux généralement associés au droit pénal : la dissuasion et l'équité des peines. L'auteur conclut que les «guidelines » (lignes directrices correspondant à des fourchettes de peine) permettent de combiner à la fois l'effort de dissuasion et le souci d'équité : une plus grande sévérité des peines imposée par le barème favorise la dissuasion en réduisant l'intervalle au sein duquel le juge peut fixer la sanction tout en lui laissant une marge de manœuvre pour tenir compte des spécificités de l'affaire. En d'autres termes, 
dissuasion et pouvoir discrétionnaire ne sont pas incompatibles dès lors que la poursuite de l'objectif de dissuasion ne contraint pas trop fortement les juges et leur laisse une possibilité d'ajustement. On retrouve la même idée en matière prud'homale dans le cas du barème mis en place en France en matière de licenciement sans cause réelle ni sérieuse. Le caractère impératif de celui-ci encourage la négociation en permettant aux parties de mieux anticiper le jugement et cadre les décisions des juges dans un intervalle que le législateur juge socialement satisfaisant ; dans le même temps, la fourchette d'indemnités prévue par ce même barème permet au juge d'ajuster sa décision aux spécificités de l'affaire en lui permettant de décider au sein d'un intervalle sans lui imposer un montant unique.

L'instauration d'un barème, surtout s'il est doté d'un caractère impératif, a pour conséquence immédiate de contraindre la décision des juges et donc de limiter leur souveraineté décisionnelle. Ceci peut être ressenti comme une perte de pouvoir qui va engendrer des réactions différentes selon les juges.

\subsubsection{Perte de motivation, résistance ou coopération}

Plusieurs auteurs se sont plus particulièrement intéressés aux motivations des juges à exercer leur activité et certains insistent d'une part sur les différentes dimensions de leur pouvoir, d'autre part sur l'alignement des sanctions sur leurs préférences. Pour mieux comprendre l'impact du barème, il est alors utile de voir quels sont les éléments identifiés dans la littérature en économie du droit qui entrent dans la fonction d'utilité des juges c'est-à-dire dans la valeur qu'ils donnent à leur travail. Ainsi, Landes et Posner (1980), dans le cadre du système jurisprudentiel de la common law nord-américaine, montrent que les juges, face à l'absence de reconnaissance salariale, cherchent plutôt à maximiser leur pouvoir entendu comme le nombre de fois où ils sont cités par d'autres juges. Posner (1996) suppose également que l'utilité des juges fédéraux dépend du revenu, du pouvoir, du prestige, des loisirs et de l'excitation intellectuelle. Selon Kornhauser (2000), les juges sont sensibles au respect de la loi, à leur pouvoir, au fait d'être cités, à leur réputation, et à leur charge de travail. De même, Higgins et Rubin (1980) estiment que les juges ont des préférences en matière de pouvoir discrétionnaire mais aussi de richesse au sens où rendre des décisions qui ne sont pas renversées augmente leurs chances de promotion dans une juridiction supérieure. Enfin, Reinganum (2000) suppose que les juges ont des préférences par rapport à la peine infligée à l'accusé : les juges auraient en tête une peine idéale et préfèreraient les peines les plus proches de celle-ci. Dans le même ordre d'idées, Miceli (2007) rappelle que si le pouvoir 
discrétionnaire des juges leur permet d'adapter leur jugement aux caractéristiques particulières de l'affaire, il leur permet aussi de prendre des décisions conformes à leurs propres préférences. La question est alors de savoir si les barèmes peuvent affecter la satisfaction des juges au travail, d'une part parce qu'ils limitent leur pouvoir discrétionnaire mais aussi parce qu'ils peuvent imposer des peines différentes de la peine considérée par le juge comme idéale pour l'affaire en question.

Boylan (2004) s'est plus particulièrement intéressé aux conséquences de l'instauration de barèmes sur les choix d'activité des juges américains. Il propose d'étudier les choix d'activité des juges fédéraux en âge de prendre leur retraite, ceux-ci pouvant opter pour continuer une activité à plein temps, prendre un emploi de juge sénior représentant une charge de travail de $25 \%$ par rapport à celle d'un juge à temps plein pour un salaire identique ou prendre leur retraite $^{15}$. Il pose 1 'hypothèse que si les barèmes réduisent la satisfaction des juges au travail, le nombre de juges admissibles à la retraite qui choisissent de continuer à exercer à plein temps devrait diminuer avec l'instauration des « guidelines » au niveau fédéral. Il explique que les «guidelines » ont notamment réduit le pouvoir des juges de district américains avec des peines plus élevées que celles qu'ils considéraient comme idéales et accru le nombre de décisions susceptibles d'être portées en appel. Les lignes directrices seraient donc à l'origine de deux sources d'insatisfaction pour les juges ${ }^{16}$. L'étude de Boylan permet en définitive de considérer que les lignes directrices ont incité les juges à choisir plus rapidement un statut de juge senior : plus précisément, les juges des tribunaux de district choisissent de devenir senior en moyenne 0,4 ans après qu'ils soient devenus éligibles, contre trois avant l'instauration des « guidelines ».

Pour leur part, Alexander et al. (1999) s'interrogent de manière plus générale sur la réaction des juges face à l'instauration de barèmes. Ils distinguent deux comportements possibles : d'une part, la résistance (les juges continuent de décider en fonction de leurs préférences) et, d'autre part, la coopération qui peut être stratégique (accepter d'appliquer une réforme pour en éviter d'autres non-souhaitées dans le futur) ou résulter d'effets d'apprentissage (évolution des préférences des juges) ou de changements de normes (les juges appliquent les barèmes car ils correspondent à des changements qu'ils souhaitent eux-mêmes). Leurs résultats confortent

\footnotetext{
${ }^{15}$ Le fait que certains juges restent à temps plein tend à montrer que la satisfaction des juges ne passe pas seulement par leur rémunération mais par d'autres éléments.

${ }^{16}$ Selon Boylan (2004), les juges peuvent ne pas apprécier les « guidelines » pour deux raisons différentes : les juges peuvent ne pas apprécier la perte de souveraineté décisionnelle en raison du cadre fixé par le barème, mais ils peuvent aussi ne pas être en accord avec les jugements prévus par le barème (peines excessives par exemple par rapport à la peine qu'ils estiment plus appropriée).
} 
le modèle coopératif (stratégie, apprentissages, nouvelles normes) car, d'une part, les amendes et les sanctions totales (amendes barémisées et autres sanctions) ont augmenté significativement avec les «guidelines » et, d'autre part, les sanctions totales tendent à être plus élevées même quand le juge n'est pas contraint (notamment quand le caractère impératif du barème s'assouplit). D'autres études soulignent que la mise en place d'un barème peut même être appréciée par certains magistrats en leur permettant de traiter plus rapidement les affaires simples et ainsi de disposer de plus de disponibilités pour traiter les affaires complexes ${ }^{17}$.

Nous nous sommes intéressés à l'impact incitatif du barème, d'une part du côté des justiciables et de leur incitation à régler leur litige à l'amiable et d'autre part du côté des juges et de leur incitation à exercer leur activité. Sur le premier point, la littérature existante apporte des réponses plutôt nuancées. Quant au second point, la littérature met en évidence la diversité des réactions des juges. Il s'agit à présent d'examiner le rôle normatif du barème, entendu comme l'influence du barème sur la mise en œuvre de la norme juridique par les juges.

\section{Barèmes et décisions des juges : le rôle normatif du barème}

Nous proposons ici de traiter de l'impact du barème sur l'activité d'interprétation juridique, vue par Deffains et Ferey (2012) comme «l'ensemble des questions relatives aux modalités d'application d'un mot, d'une phrase ou d'un instrument dans un certain contexte ». Si l'on considère le barème comme un outil d'aide à la décision à disposition du juge, la question est de savoir dans quelle mesure cet instrument contraint, conditionne, oriente, ou seulement influence la décision du juge, selon que le barème est impératif ou indicatif et qu'il fournit des intervalles ou des nombres. Les objectifs poursuivis derrière cet encadrement de la décision judiciaire et donc cette limitation de l'interprétation de la norme par le juge peuvent être multiples. Il peut s'agir d'une part de limiter l'interprétation du juge pour garantir une plus grande équité, horizontale et/ou verticale des décisions (4.1); il peut s'agir aussi d'aligner les décisions des juges sur les préférences sociales dans un but d'efficacité (4.2). Dans les deux cas, le barème tend à limiter l'influence des préférences individuelles ou à éviter les effets non

\footnotetext{
${ }^{17}$ Ainsi, par exemple une enquête qualitative récente réalisée auprès des juges aux affaires familiales français (Jeandidier et Ray, 2019) montre qu'un barème pourrait accroître la satisfaction des juges au travail en leur permettant dans le cas des pensions alimentaires pour enfants de dégager du temps pour régler le plus harmonieusement possible la question du partage de l'hébergement de l'enfant dans les divorces conflictuels.
} 
désirés résultant de biais cognitifs éventuels. Ceci étant, comme nous allons le montrer, le pouvoir d'un barème de réduire la décision des juges à un acte de pure connaissance est dans une certaine mesure limité.

\subsection{Limiter le pouvoir interprétatif des juges pour plus d'équité}

Par construction, un barème traite également des égaux. Ce faisant, sa stricte application devrait conduire à éliminer la disparité des décisions inter-juges. De nombreux travaux ont permis d'évaluer dans quelle mesure la diffusion des barèmes dans les juridictions s'est accompagnée de gains en termes d'équité horizontale (4.1.1). Parallèlement, d'autres travaux ont soulevé la question de la pertinence des critères retenus pour définir les situations comparables, conduisant à discuter des gains en termes d'équité verticale associés à la mise en place des barèmes (4.1.2).

\subsubsection{Un gain d'équité horizontale discuté}

D'après les travaux empiriques réalisés sur le sujet, la capacité des barèmes à renforcer l'équité horizontale semble toute relative en ce sens que la mise en place de barème a tout au plus permis de contenir la disparité des décisions (4.1.1.1) et n'a pas permis d'éradiquer les prises de décisions biaisées (4.1.1.2).

\subsubsection{Une variabilité des décisions seulement atténuée}

L'une des raisons à l'instauration de barèmes réside dans la volonté des décideurs publics de réduire la disparité des décisions pour des affaires identiques, dans un souci d'équité horizontale. Au regard des études sur le sujet, il semblerait que la capacité des barèmes à remplir cet objectif soit limitée. Par exemple, dans le domaine pénal, à partir de l'exploitation de données de justice, Anderson et al. (1999) constatent que la disparité des peines imputables au juge a relativement diminué après la mise en place de barèmes impératifs au milieu des années 1980. La différence attendue dans la durée des peines de deux juges ayant des dossiers comparables était de 16 à $18 \%$ dans la période précédant la réforme, mais seulement de 8 à $13 \%$ dans la période post barème. Les travaux de Hofer, Blackwell et Ruback (1999) portant sur des données de même type aboutissent à une conclusion similaire en montrant que la mise en place des barèmes aurait permis de réduire la disparité entre les juges, du moins pour certains types d'infractions et dans certains seulement des districts examinés. Ces résultats 
s'observent également dans le domaine des affaires civiles. En utilisant les données de la National Longitudinal Survey of Labor market Experience of Youth, Argys et al. (2001) montrent que l'introduction des barèmes en matière de pension alimentaire aurait réduit de manière significative la disparité des montants de pension alimentaire entre cas similaires, principalement par la réduction de la probabilité des montants extrêmes.

A l'inverse, d'autres études concluent que la disparité inter-juges aurait augmenté dans la période suivant l'adoption des barèmes, sans pour autant avancer d'arguments explicatifs à ce résultat (Waldfogel, 1991 ; Payne, 1997 ; Lacasse et Payne, 1999). Par exemple, Lacasse et Payne (1999) ont examiné si la variabilité des peines d'emprisonnement attribuable aux juges avait été éliminée par l'utilisation des barèmes. Leur étude montre que, une fois pris en compte les biais de sélection liés aux caractéristiques des affaires, des juges et des accusés, cette variabilité aurait augmenté. Plus précisément, le montant de la variation attribuable au juge dans les décisions de première instance qui était de $4 \%$ avant la réforme serait comprise entre 5 et $13 \%$ après la réforme selon le type de crime et de tribunal. Et les auteurs de conclure à l'incapacité des barèmes à éliminer toute variabilité des sanctions entre les juges, notamment en raison de la possibilité de ces derniers de conserver une marge d'appréciation même en présence de barèmes obligatoires. Dans le cas de cette étude, les auteurs estiment que les juges peuvent effectivement être sensibles aux remords de l'accusé ou peuvent apprécier les preuves différemment ${ }^{18}$.

Plus récemment, et sur données expérimentales, une étude française aboutit à des résultats convergents à ceux mis en évidence dans le cas américain. S'appuyant sur la soumission d'une cinquantaine de cas types aux élèves de première année de l'Ecole Nationale de la Magistrature, Jeandidier et Ray (2019) montrent qu'en moyenne, on observerait un effet de réduction de la disparité des décisions (i.e. la variance intra-cas type est plus faible lorsque les auditeurs ont l'opportunité de recourir au barème) mais que cet effet ne serait pas systématique, un accroissement de l'hétérogénéité étant observé pour certains cas types ${ }^{19}$.

\footnotetext{
${ }^{18}$ En matière pénale, les barèmes américains consistent à proposer des fourchettes de peines par catégorie d'infraction, en fonction des caractéristiques de l'affaire (c'est-à-dire les antécédents criminels du délinquant et la gravité de l'infraction actuelle). Jusqu'en 2005, date à laquelle le caractère impératif des barèmes américains a été assoupli, les juges n'étaient autorisés à s'écarter de cette fourchette (vers le bas ou vers le haut) que dans certaines circonstances et sous réserve de donner des raisons pertinentes liées à des caractéristiques de l'affaire ou à des questions juridiques (Schanzenbach et Tiller, 2007).

${ }^{19}$ Les auteurs observent un effet d'homogénéisation lorsque les élèves magistrats traitent des affaires où les montants de pension alimentaire offerts par le débiteur et demandé par le créancier sont très différents ou des affaires avec un couple de revenus parentaux inégaux en faveur de la mère; à l'inverse, il augmenterait l'hétérogénéité dans les affaires où les couples de revenus sont inégaux au profit du père. Dans ce dernier cas, le montant de pension alimentaire proposé par le barème pouvant être supérieur à la demande de la créancière,
} 


\subsubsection{Des juges à la partialité contenue mais non supprimée}

D'autres travaux, complémentaires aux premiers, ont cherché à vérifier si l'introduction de barèmes permettait d'éliminer l'influence de certains facteurs extra-légaux, sources d'iniquité. Il semblerait que si les barèmes impératifs permettent de contenir la partialité des juges, ils ne parviennent pas à empêcher totalement ces derniers de prendre des décisions en fonction des caractéristiques personnelles des défendeurs alors même que celles-ci ne sont pas incluses dans les critères de décision pris en compte par le barème, voire sont prohibées. Ainsi, les travaux réalisés sur le cas américain après l'instauration de barèmes obligatoires dans le domaine pénal ont mis en évidence le maintien de disparités dans les décisions selon l'origine ethnique, le genre, l'éducation ou le niveau socio-économique des délinquants. C'est par exemple ce qu'illustre l'étude de Sorensen et al. (2012). Ces derniers s'intéressent aux décisions des juges américains contraints de se soumettre à la fourchette de peines du barème au risque sinon de s'exposer à des recours. Ils développent un modèle théorique où le juge cherche à maximiser une fonction d'utilité par rapport à une sentence idéale, sous contrainte de coûts. La sentence optimale, qui maximise l'utilité du juge, est ainsi fonction de la sentence idéale (qui dépend des caractéristiques de l'affaire et du défendeur) mais aussi du fait que le juge s'éloigne plus ou moins des sentences minimale et maximale du barème. Les auteurs cherchent alors à estimer dans quelle mesure l'écart entre les sentences attendues moyennes entre deux groupes (définis par l'origine ethnique ou le genre) s'explique par les caractéristiques des affaires, le résidu étant supposé résulter des préférences des juges. Leurs résultats empiriques montrent que, toutes choses égales par ailleurs (c'est-à-dire en contrôlant les critères retenus par les barèmes, à savoir la gravité de l'infraction et les antécédents criminels du délinquant), les juges sanctionnent moins sévèrement les femmes et plus sévèrement les hommes noirs, relativement aux hommes blancs.

Suite à une décision de la Cour Suprême américaine en 2005, le caractère impératif des barèmes en matière pénale a été relâché. L'analyse de l'impact de cette réforme a permis de montrer que même si les barèmes ne permettaient pas d'éradiquer la partialité des juges, ils permettaient à tout le moins de la contenir lorsque ces derniers avaient un caractère impératif. En effet, les travaux ont montré que la réforme avait conduit à un renforcement de l'influence des facteurs extra légaux dans les décisions des juges, les travaux convergeant notamment sur

certains auditeurs ont retenu le montant issu du barème tandis que d'autres ont préféré respecter la règle procédurale conduisant à ne pas fixer une pension alimentaire supérieure au montant demandé. L'écart de comportement entre auditeurs contribue à expliquer l'accroissement de la disparité des décisions dans ce cas. 
le constat d'un accroissement des différences de peines entre noirs et blancs par rapport à la période précédente où les barèmes étaient obligatoires (Fischman et Schanzenbach 2012, Rehavi et Starr, 2014 ; Yang 2015).

Le point commun de ces différentes études est de fournir assez peu d'explications sur les causes de ces disparités de jugement. On peut néanmoins vraisemblablement penser que ces disparités sont en partie le résultat des préférences individuelles des juges qui seraient relativement partagées au sein de la profession et qui se traduiraient par des différences de jugement statistiquement significatives (Gennaioli et Shleifer, 2008). On peut aussi considérer que les juges sont, comme les autres agents économiques, victimes de biais cognitifs et qu'ils commettent des erreurs indépendantes de leur orientation politique ou de la recherche de leur intérêt personnel (Guthrie et al., 2001 ; Wistrich et al., 2015 ; Spamann et Klöhn, 2016 ; Liu, 2018 ; Kahan, 2015). La partialité relative de leurs décisions résulterait d'heuristiques (de confirmation $^{20}$, d'ancrage ${ }^{21}$ ou de disponibilité ${ }^{22}$ ), que le barème ne suffirait pas à corriger.

\subsubsection{Un gain d'équité verticale contesté}

Un barème formalise les situations jugées comme comparables devant donner lieu à la fixation d'un montant identique. Certains travaux remettent en cause les critères retenus pour déterminer ces situations comparables en soulignant leur nombre trop limité, conduisant à la fixation de montants insuffisamment proportionnés ${ }^{23}$.

Selon Waldfogel (1998), les barèmes conduiraient à une uniformité excessive des décisions, affectant la proportionnalité entre délit et sanction. L'objectif des barèmes est en effet de réduire la disparité injustifiée en limitant la variabilité des sanctions. Mais ce faisant, ils peuvent nuire à la proportionnalité entre le délit et la sanction ou empêcher d'appliquer une sanction qui correspond mieux au délit. L'étude empirique menée par l'auteur montre que les réductions dans la disparité injustifiée permises par le barème ne compensent pas la perte en termes de proportionnalité, venant en cela nuancer les arguments en faveur de l'instauration d'un barème. Les barèmes élimineraient donc, selon Waldfofgel à la fois la bonne disparité (c'est-à-dire celle liée à l'hétérogénéité des affaires) et la mauvaise disparité (c'est-à-dire

\footnotetext{
${ }^{20}$ Les juges accorderaient plus de poids aux informations qui confirment leurs croyances qu'à celles qui les contredisent.

${ }^{21}$ Les juges auraient une valeur de référence qui constituerait un point de repère, d'ancrage, de leurs décisions futures.

${ }^{22}$ Les décisions des juges sur une affaire précise seraient influencées par des exemples d'affaires antérieures.

${ }^{23} \mathrm{Ce}$ nombre insuffisant de critères est par exemple l'une des critiques adressées par certains juges à l'égard de l'actuel barème en matière de CEEE. Selon ces derniers, ceci rendrait difficile le recours à cet outil dans certains cas d'espèces, voire remettrait en cause l'usage même de cet outil (Bourreau-Dubois et al., 2019).
} 
celles liées à la subjectivité décisionnaire des juges, susceptibles d'être influencés par des facteurs extra-légaux), sauf à ce qu'ils intègrent toutes les caractéristiques de l'affaire comme celles du mis en cause, pertinentes pour établir la sanction. En d'autres termes, le problème des barèmes serait l'uniformité, pas la variabilité (Schulhofer, 1992).

Cette réserve est aussi énoncée dans le cas des pensions alimentaires ou des indemnités prud'homales. Ainsi, dans le premier cas, plusieurs études ont souligné que les barèmes pouvaient être perçus comme source d'iniquité par les parties notamment parce qu'ils ne permettaient pas de prendre en compte le caractère évolutif des situations et de la complexité des histoires familiales (Melli, 2000 ; Oldham, 2000). Dans le second, le risque de perte de proportionnalité a été évoqué lors des discussions qui ont eu lieu en amont de la mise en place en France du barème d'indemnités prud'homales. Le barème impératif instauré par l'article 2 de l'Ordonnance n²017-1387 du 22 septembre 2017 a conduit à la suppression de la distinction entre «licenciement sans cause réelle ni sérieuse » et « licenciement abusif » et à fixer un barème proposant une indemnité plancher et plafond pour l'ensemble des salariés concernés ${ }^{24}$. Ce plafond conduit de fait à une uniformisation des indemnités par une réduction de l'étendue des indemnités possibles ${ }^{25}$.

La volonté du législateur de limiter le pouvoir interprétatif des juges par l'instauration de barèmes peut se justifier au regard de l'équité mais aussi de l'efficacité.

\subsection{Limiter l'interprétation pour plus d'efficacité}

L'efficacité du barème peut être évaluée à partir de sa capacité à créer les incitations dans un objectif de minimisation du coût social ou de maximisation du bien-être collectif. Ainsi, l'objectif peut être par exemple de garantir le paiement des pensions alimentaires pour minimiser le coût social des divorces (notamment en termes de risques de pauvreté) ou de mettre en place un système de sanctions visant à minimiser le coût social des activités illégales. Le législateur peut alors concevoir le barème comme un instrument de politique publique, cadrant les décisions des juges dans le sens souhaité. A titre d'illustration, en

\footnotetext{
${ }^{24}$ Avant cette réforme, le droit du travail distinguait deux cas de figure. En cas de licenciement sans cause réelle et sérieuse, c'est-à-dire lorsque le salarié avait plus de 2 ans d'ancienneté dans une entreprise comptant plus de 11 salariés, il était prévu un plancher en matière d'indemnité (6 mois de salaire) mais pas de plafond. En cas licenciement abusif, c'est-à-dire lorsque les deux conditions précédentes n'étaient pas respectées, le montant d'indemnités n'était pas encadré et il revenait au salarié de chiffrer son préjudice.

${ }^{25}$ Comme le notent Desrieux et Espinosa (2019), on peut penser que ce barème incitera probablement les demandeurs à augmenter les montants demandés et attribués au titre des autres chefs de demande (ex : indemnité compensatrice de préavis ou de congés payés), voire à invoquer d'autres motifs à titre complémentaire voire principal. Un moyen de sortir de l'uniformité jugée injuste par le demandeur consiste en effet pour celui-ci à déplacer une partie de ses prétentions dans un espace du droit où le juge retrouve son pouvoir discrétionnaire.
} 
matière de pension alimentaire, la création de barèmes aux Etats-Unis visait principalement à apporter une réponse au problème de la pauvreté des familles monoparentales, dont l'origine pour certains auteurs était la fixation par les juges de montants très (trop) faibles (Garfinkel et Oellerich, 1989 ; Garrison, 2000). En imposant des montants plus élevés, il s'agissait de relever le niveau de vie des enfants vivant dans une famille monoparentale. Cependant, la plupart des travaux montrent que l'objectif n'a été que partiellement atteint, notamment en raison de l'homogamie sociale: les ex-conjoints des mères de familles monoparentales pauvres ayant très souvent de faibles revenus, les montants de pension alimentaire perçus par les familles monoparentales, même relevés du fait du barème, restent modestes (Hu et Meyer, 2003). D'autre part, le taux d'effectivité des versements de pensions alimentaires est resté relativement faible aux Etats-Unis, ce phénomène pouvant s'expliquer par des effets désincitatifs inhérents aux barèmes (ou à certains types de barèmes). Ainsi certaines études (Bassi et Lerman, 1996 ; Chien-Chung et alii, 2005 ; Roff, 2010) montrent que «taxer » trop lourdement les parents non gardiens à faibles revenus ne serait pas très efficace au sens où cela pourrait faire basculer certains d'entre eux dans la pauvreté et les décourager à verser une pension, même insuffisante. Plus récemment, les travaux français de Jeandidier et Ray (2019) menés sur des données expérimentales collectées à l'Ecole Nationale de la Magistrature, montrent que le recours au barème en matière de pension alimentaire aurait pour effet de n'augmenter que modestement le montant de pension alimentaire fixé par les auditeurs ${ }^{26}$.

En matière pénale, l'un des objectifs des barèmes aux Etats-Unis était de renforcer la dissuasion à commettre des crimes/délits, non pas directement par l'instauration de barèmes visant à l'uniformité, mais indirectement par le fait que les barèmes obligeaient les juges à fixer des sanctions plus sévères. Là encore, les résultats des études ne convergent pas. Ainsi, si Freeborn et Hartmann (2010) montrent qu'à la suite d'un amendement prononcé en 2003 contraignant les juges fédéraux à ne pas sortir de la fourchette proposée par le barème, on observe bien un relèvement des peines, ce fait n'est pas confirmé par l'ensemble des travaux. Ainsi, selon Andreoni (1991), les jurys auraient peur de se tromper et de condamner un innocent de sorte que plus les peines sont sévères et moins ils condamnent. Des sanctions plus sévères (peines planchers) conduiraient à moins de condamnations et aurait donc un effet dissuasif plus faible. Lacasse et Payne (1999, op. cit.) proposent, pour leur part, une étude empirique sur deux tribunaux, visant à tester l'impact des «guidelines » notamment sur la durée des peines. Ils concluent qu'il n'est pas certain que la durée des peines ait augmenté.

\footnotetext{
${ }^{26}$ Ce résultat pourrait tenir à une spécificité du droit français. En effet, les juges sont contraints par les propositions des parties, qui peuvent être inférieures aux montants proposés par le barème indicatif.
} 
Plus précisément, ils observent que la durée des peines de prison a bien augmenté lorsqu'il y a procès mais qu'il n'y a pas d'augmentation systématique équivalente dans les affaires ayant donné lieu à un plaider-coupable. L'effet dissuasif du barème pourrait donc s'en trouver amoindri. Parker et Atkins (1999) aboutissent à la même conclusion après avoir montré empiriquement que les «guidelines » à caractère incitatif mises en place en matière de droit pénal des affaires (amendes), n'auraient pas eu d'impact sur le niveau moyen des sanctions et leur variabilité, ni sur la structure des peines (relation entre le dommage et la sanction).

Un moyen de pallier la faible effectivité des barèmes en matière de dissuasion pourrait consister à associer la sanction prévue par les barèmes à des sanctions complémentaires, laissant au juge une partie de son pouvoir discrétionnaire. C'est ce que proposent Kahan et Posner (1999) dans le cas de la criminalité en col blanc. Selon ces derniers il faudrait coupler des sanctions non monétaires fondées sur la réputation (honte) aux «guidelines ». Ils montrent que ce type de sanction serait compatible avec l'objectif d'uniformité inhérent aux lignes directrices tout en préservant le caractère dissuasif des sanctions. L'instauration de « guidelines » pour ce type de délits a en effet conduit à substituer des peines de prison aux amendes, sans effet sur la dissuasion. Les auteurs défendent alors l'idée que les lignes directrices peuvent conserver leur utilité, à condition de coupler un autre système de sanction reconnu pour son caractère dissuasif. La sanction « complète » reposerait sur deux éléments : une composante réputationnelle (fondée sur la honte) qui consisterait en une publicité stigmatisante sous la forme d'une annonce dans les médias, payée par le défendeur et détaillant la nature de l'infraction commise, la nature de la peine infligée et les mesures prises pour empêcher la répétition d'infractions similaires, et une composante monétaire sous forme d'amende (sauf dans les cas les plus graves où la prison s'impose) qui varierait en fonction de la gravité du crime, conformément aux critères énoncés dans les lignes directrices. Ce type de sanctions n'introduirait en outre pas de tension entre des sanctions réputationnelles, plutôt individualisées, et l'objectif d'uniformité des peines poursuivis par le barème en vertu de l'équité horizontale. Les sanctions réputationnelles sont effectivement exposées à un risque élevé de disparité entre juges car elles reposent sur leur appréciation des faits et du contexte et donc laissent une grande latitude à leur pouvoir discrétionnaire, sauf si elles consistent en des sanctions de type «publicité stigmatisante » où le pouvoir discrétionnaire du juge se limite à décider du média et du format de la publicité, comme le préconisent les auteurs. Ici, la justification de la sanction réputationnelle repose sur son caractère stigmatisant et donc dissuasif, identique à celui de l'emprisonnement (au moins pour les cas les moins graves) 
mais permettant une économie de coûts à la société. Elle viendrait ainsi utilement compléter l'amende qui est souvent perçue par le délinquant comme le prix à payer pour son comportement et ne présente donc pas le même effet dissuasif. En d'autres termes, généraliser l'idée de Kahan et Posner reviendrait à accepter que, si l'objectif du barème est l'uniformisation des décisions en vertu de l'équité horizontale, l'objectif de dissuasion et plus généralement d'efficacité peut conduire à lui associer d'autres décisions qui laissent de la place au pouvoir discrétionnaire du juge. De cette manière, il semble possible de préserver la bonne disparité (inter-affaires), tout en réduisant la mauvaise (inter-juges).

L'ensemble de ces résultats permet de mettre en évidence la faible capacité des barèmes à gommer toutes les sources d'iniquité, y compris les plus subjectives et les plus discutables mais aussi à atteindre les objectifs d'efficacité. En conséquence, même en présence de barèmes qui pourraient transformer l'interprétation juridique en simple acte de connaissance (celle-ci se limitant à appliquer une règle de calcul sur la base d'éléments préalablement recensés) où prévaudrait neutralité et impartialité, l'acte de juger paraît rester au moins en partie un acte de volonté, ce qui peut avoir des conséquences aussi bien en termes d'équité que d'efficacité. En d'autres termes, un juge rationnel au sens économique pourrait continuer de juger en vertu de ses préférences individuelles ${ }^{27}$ tandis que les décisions des juges imparfaitement rationnels seraient toujours affectées par des biais cognitifs, seulement partiellement corrigés par le barème ${ }^{28}$.

\section{CONCLUSION}

Depuis 2010, des barèmes ont fait leur apparition dans le paysage judiciaire français, tant en matière de pensions alimentaires pour enfants (mise en circulation par le Ministère de la Justice en 2010 de la table de référence indicative pour calculer une Contribution pour l'entretien et l'éducation de l'enfant) que plus récemment en matière prud'homale (ordonnance relative à la prévisibilité et la sécurisation des relations de travail du 22 septembre 2017). Dans d'autres domaines, leur éventuelle mise en place suscite des débats récurrents, notamment en matière de dommage corporel. Dans ce contexte de barémisation accrue de la justice, notre analyse a pour ambition d'étudier ce qui est commun à leur application, quel que soit le domaine du droit considéré, tout en admettant que chaque

\footnotetext{
${ }^{27}$ La conformité des décisions aux sentences prévues par le barème pourrait en outre dépendre de l'expérience des juges, les écarts par rapport aux jugements prévus par le barème étant d'autant plus faibles que le type d'affaires est fréquent ou que le juge est spécialisé dans le domaine (Bushway et al., 2012).

${ }^{28}$ La question du rôle et des modalités du redressement cognitif et de son calibrage adéquat reste donc posée.
} 
domaine a ses spécificités propres, justifiant parfois des freins, voire des oppositions, à leur mise en œuvre réglementaire (Sayn, 2014).

Dans ce cadre, l'instauration de barèmes, en France comme aux Etats-Unis, est justifiée à la fois par des objectifs d'efficacité (améliorer le taux de règlement amiable, accroître la productivité des juges) et d'équité (verticale et horizontale). Dans cette perspective, il est attendu des barèmes un impact sur le comportement des justiciables et des juges, plus ou moins incités à s'investir dans la résolution du conflit (par la recherche d'une résolution amiable pour les justiciables, par une adhésion plus ou moins forte au barème pour résoudre le litige dans le cas des juges). Au-delà des aspects incitatifs, les barèmes ont également une dimension interprétative puisqu'ils indiquent, avec plus ou moins de latitude, quelle doit être la décision des juges. Il s'agit ici principalement de réduire la part subjective de la décision judiciaire. Or, les juges s'opposent souvent au barème au motif qu'il faut tenir compte le plus finement possible des spécificités de l'affaire. La régulation du système juridique est donc soumise à une possible tension entre d'un côté la nécessité de laisser de la liberté au juge pour coller aux spécificités de l'affaire (conduisant à une disparité inter-affaires, liées aux spécificités de chacune) et de l'autre, le souhait de réduire le pouvoir discrétionnaire des juges pour limiter l'expression des préférences personnelles du juge et/ou l'éventuel impact de biais cognitifs (conduisant à limiter la disparité inter-juges à affaire donnée). Une question plus large découle de l'analyse limitée aux seuls barèmes : quels sont les outils d'aide à la décision à disposition des juges les mieux à même de limiter cette tension entre une «bonne » (interaffaires) et une «mauvaise» (inter-juges) disparité ? Si l'on se place du côté de l'individualisation des décisions, la meilleure façon d'atteindre cet objectif consiste certainement à laisser le juge décider seul sur la base des pièces disponibles. De ce point de vue, l'usage d'algorithmes, dans une optique de justice dite « prédictive », permettrait d'affiner la décision du juge en lui permettant de s'appuyer sur des précédents similaires et ce d'autant plus finement que les données disponibles sont riches et correctement traitées. A l'inverse, le barème, dès lors qu'il est construit, conduit pour sa part à limiter cette individualisation de manière mécanique car reposant sur la constitution de catégories homogénéisant les spécificités individuelles. Si l'on se place désormais du côté de la subjectivité de la décision judiciaire, le barème est probablement l'instrument qui permet le mieux d'écarter cette source de disparité entre les décisions, même s'il n'y parvient pas totalement. A l'inverse, les algorithmes, parce qu'ils sont fondés sur les décisions passées, auront plutôt tendance à reproduire la subjectivité liée aux préférences ou aux biais des juges. 
Sous cet angle, le barème pourrait apparaître comme un compromis satisfaisant : il limite le caractère subjectif de la décision judiciaire tout en préservant la prise en compte des spécificités de l'affaire, et ce d'autant plus qu'il intègre un grand nombre d'éléments. Mais l'avantage principal du barème réside certainement dans la capacité du décideur public à le construire, de sorte qu'il repose sur des choix (fondés sur des raisonnements juridiques, économiques, démographiques, politiques,... toujours discutables mais justifiables), alors que l'application de l'intelligence artificielle au big data de la justice tend plutôt à généraliser des barèmes constatés, au risque de reproduire des biais non souhaités (que les barèmes construits se proposent de combattre) d'une part et de cristalliser la jurisprudence d'autre part.

Tout aussi efficace et équitable que soit le barème, comme nous l'avons montré, un paramètre d'incertitude demeurera cependant toujours : la volonté dont témoignent les magistrats de défendre leur liberté d'appréciation et de décision. Les barèmes posent donc incontestablement la question de ce que la société attend de ses juges, dans un monde où les outils d'aide à la décision se multiplient et interrogent le judiciaire sur sa plus-value décisionnelle. 


\section{Bibliographie}

Allen D. W., Brinig M. [2012], Child support guidelines and divorce incentives, International Review of Law and Economics, 32, 309-316.

Ancelot L., Doriat-Duban M. [2010], Analyse économique du plaider coupable, Revue économique, 61(2), 237-261.

Anderson J. T., Kling J. R., Stith K. [1999], Measuring Inter-judge Sentencing Disparity: Before and After the Federal Sentencing Guidelines, Journal of Law and Economics, 42(1), 271-307.

Andreoni J. [1991], Reasonable Doubt and the Optimal Magnitude of Fines: Should the Penalty Fit the Crime?, RAND Journal of Economics, 22, 385-95.

Argys L. M., Peters H. E., Waldman D. M. [2001], Can the Family Support Act Put Some Life Back Into Deadbeat Dads? An Analysis of Child-Support Guidelines, Award Rates, and Levels, The Journal of Human Resources, 36(2), 226-252.

Alexander C. R., Arlen J., Cohen M. A. [1999], Regulating Corporate Criminal Sanctions: Federal Guidelines and the Sentencing of Public Firms, The Journal of Law \& Economics, 42(S1), 393-422.

Barfeld J. [2000], Child Support and Post-divorce Economic Well-Being of Mothers, Fathers and Children, Demography, 37(2), 203-213.

Barthélémy J., Cette G. [2015], Réformer le droit du travail, Paris, Editions Odile Jacob.

Bassi L. J., Lerman R. I. [1996], Reducing the Child Support Welfare Disincentive Problem, Journal of Policy Analysis and Management, 15(1), 89-96.

Becker G. [1968], Crime and punishment: An economic approach, Journal of Political Economy, 76(2), 169-217.

Bourreau-Dubois C., Deffains B., Desrieux C., Doriat-Duban M., Espinosa R., Jeandidier B., Mansuy J., Ray J.-C. [2019], La barémisation de la justice : une approche par l'analyse économique du droit, Paris, Mission de Recherche Droit et Justice.

Boylan R. T. [2004], Do the Sentencing Guidelines Influence the Retirement Decisions of Federal Judges?, The Journal of Legal Studies, 33(1), 231-253. 
Bushway S. D., Owens E. G., Piehl A. M. [2012], Sentencing Guidelines and Judicial Discretion: Quasi-Experimental, Evidence from Human Calculation Errors, Journal of Empirical Legal Studies, 9(2), 291-319.

Chien-Chung H., Mincy R. B., Garfinkel I. [2005], Child Support Obligations and LowIncome Fathers, Journal of Marriage and Family, 67(5), 1213-1225.

Deffains B., Langlais E. [2006], Incentives to cooperate and the discretionary power of courts in divorce law, Review of Economic Household, 4, 423-439.

Deffains B., Ferey S. [2012], L'économie du droit entre révolution cognitive et tournant réaliste, Revue Française d'Economie, 27(4), 45-80.

Desrieux C., Espinosa R. [2019], Une analyse de la disparité pré-barème: le cas du contentieux prud'homal, in : Bourreau-Dubois C., Deffains B., Desrieux C., Doriat-Duban M., Espinosa R., Jeandidier B., Mansuy J., Ray J.-C., La barémisation de la justice : une approche par l'analyse économique du droit, Paris, Mission de Recherche Droit et Justice, $31-82$.

Desrieux C., Espinosa R. [2020], La barémisation des indemnités pour licenciement abusif : quelques éléments d'analyse empirique, Revиe économique, 71, 527-556.

Ferey S., Deffains B. [2014], Les fonctions cognitives du droit dans la pensée de Cass Sunstein, Revue Economique, 2(65), 263-278.

Fischman J. B., Schanzenbach M. M. [2012], Racial Disparities under the Federal Sentencing Guidelines: The Role of Judicial Discretion and Mandatory Minimums", Journal of Empirical Legal Studies, 9(4), 729-64.Freeborn B. A., Hartmann M. E. [2010], Judicial Discretion and Sentencing Behaviour: Did the Feeny Amendment rein in District Judges?, Journal of empirical Legal Studies, 7(2), 355-378.

Garfinkel I., Oellerich D. [1989], Noncustodial Fathers' Ability to Pay Child Support, Demography, 26(2), 219-233.

Garrison M. [2000], The Goals and Limits of Child Support Policy, in: Oldham J. T., Melli M. S., Child Support The Next Frontier, Ann Arbor, University of Michigan Press, 16-45.

Gennaioli N., Shleifer A. [2008], Judicial Fact Discretion, Journal of Legal Studies, 37, 1-35.

Guthrie C., Rachlinski J. J., Wistrich A. J. [2001], Inside judicial mind, Cornell Law Review, $86,778-830$. 
Higgins R., Rubin P. [1980], Judicial Discretion, Journal of Legal Studies, 9, 129-38.

Hofer P. J., Blackwell K. R., Ruback R. B. [1999], The effect of Sentencing Guidelines on Inter Judge Sentencing Disparity, The Journal of Criminal Law and Criminology, 90(1), 239321.

Hu M.-C., Meyer D. R. [2003], Child Support Orders and Payments: Do Lower Orders Result in Higher Payments?, Madison, Wisconsin Department of Workforce Development, Institute for Research on Poverty.

Jeandidier B., Ray J.-C. [2019], L'analyse ex post du recours à un barème en matière de fixation de CEEE à partir d'une approche expérimentale, in : Bourreau-Dubois C., Deffains B., Desrieux C., Doriat-Duban M., Espinosa R., Jeandidier B., Mansuy J., Ray J.-C., La barémisation de la justice : une approche par l'analyse économique du droit, Paris, Mission de Recherche Droit et Justice, 85-178.

Kahan D. M., Posner E. A. [1999], Shaming White- Collar Criminals: A Proposal for Reform of the Federal Sentencing Guidelines, The Journal of Law \& Economics, 42(S1), 365-392.

Kahan D. M. [2015], Laws of Cognition and the Cognition of Law, Cognition, 135, 56-60.

Kornhauser L. A. [2000], Judicial Organization and Administration, in: Bouckaert B., De Geest G. (eds.), The Economics of Crime and Litigation, Encyclopedia of Law and Economics, Vol. 5, Northampton, Edward Elgar Pub.

Lacasse C., Payne A. A. [1999], Federal Sentencing Guidelines and Mandatory Minimum Sentences: Do Defendants Bargain the Shadow of the Judge?, The Journal of Law \& Economics, 42(S1), 245-270.

Landes W., Posner R. A. [1980], Legal Change, Judicial Behavior and the Diversity Jurisdiction, Journal of Legal Studies, 9, 1-39.

Liu Z. [2018], Does Reason Writing Reduce Decision Bias? Experimental Evidence from Judges in China, Journal of Legal Studies, 47, 83-118.

Miceli T. [2007], Criminal Sentencing Guidelines and Judicial Discretion, Contemporary Economic Policy, 26( 2), 207-215.

Melli M. S. [2000], Guideline Review: The Search for an Equitable Child Support Formula, in : Oldham J. T., Melli M. S. (eds.), Child Support The Next Frontier, Ann Arbor, University of Michigan Press, 113-127. 
Oldham J. T. [2000], New Methods to Update Child Support, in : Oldham J. T., Melli M. S. (eds.), Child Support The Next Frontier, Anne Arbor, University of Michigan Press, 128-150.

Parker J. S., Atkins R. A. [1999], Did the Corporate Criminal Sentencing Guidelines Matter? Some Preliminary Empirical Observations, The Journal of Law and Economics, 42(S1), 423454.

Payne A. [1997], Does Inter-Judge Disparity Really Matter? An Analysis of the Effects of Sentencing Reforms in Three Federal District Courts, International Review of Law and Economics, 17, 337-366.

Porchy-Simon S. [2014], L'utilisation des barèmes en droit des dommages corporels au regard des principes fondamentaux du droit de la responsabilité civile, in : Sayn I. (ed.), Le droit mis en barèmes?, Paris, Ed. Dalloz.

Posner R. A. [1996], The Federal Courts: Challenge and Reform, Cambridge, Harvard University Press.

Rehavi M. M., Starr S. B. [2014], Racial Disparity in Federal Criminal Sentences, Journal of Political Economy, 122(6), 1320-1354.

Reinganum J. F. [2000], Sentencing Guidelines, Judicial Discretion, and Plea Bargaining, The RAND Journal of Economics, 31(1), 62-81.

Roff J. [2010], Welfare, Child Support and Strategic Behavior. Do High Orders and Low Disregards Discourage Child Support Awards?, Journal of Human Resources, 45(1), 135-154.

Sayn I., Perrocheau V., Favier Y., Merley N. [2019], Les barèmes (et autres outils techniques d'aide à la décision) dans le fonctionnement de la justice, Paris, Mission de Recherche Droit et Justice.

Sayn I. [2014], Le droit mis en barèmes ?, Paris, Ed. Dalloz.

Schanzenbach M. M., Tiller E. H. [2007], Strategic Judging under the U.S. Sentencing Guidelines: Positive Political Theory and Evidence, Journal of Law, Economics, and Organization, 23(1), 24-56.

Schulhofer S. J. [1992], Assessing the Federal Sentencing Process: The Problem is Uniformity, Not Disparity, American Criminal Law Review, 29(3), 833-873.

Spamann H., Klöhn L. [2016], Justice Is Less Blind, and Less Legalistic, Than We thought: Evidence from an Experiment with Real Judges, Journal of Legal Studies, 45, 255-280. 
Sorensen T., Sarnikar S., Oaxaca R. L. [2012], Race and Gender Differences Under Federal Sentencing Guidelines, The American Economic Review, 102(3), 256-260.

Taha A. E. [2001], The equilibrium effect of legal rule changes: Are the federal sentencing guidelines being circumvented?, International Review of Law and Economics, 21, 251-269.

Yang C. S. [2015], Free at Last? Judicial Discretion and Racial Disparities in Federal Sentencing, The Journal of Legal Studies, 44(1), 75-111.

Waldfogel J. [1991], Aggregate inter-judge disparity in federal sentencing: evidence from three districts, Federal Sentencing Reporter, 4(3),151-154.

Waldfogel J. [1998], Does Inter-Judge Disparity Justify Empirically Based Sentencing Guidelines?, International Review of Law and Economics, 18, 293-304.

Wistrich A. J., Rachlinski J. J., Guthrie C. [2015], Heart Versus Head: Do Judges Follow the Law or Follow Their Feelings, Texas Law Review, 93, pp. 855-923. 